\title{
The Yang-Mills measure in the Kauffman bracket skein module
}

Doug Bullock, Charles Frohman and Joanna Kania-Bartoszynska

\begin{abstract}
For each closed, orientable surface $\Sigma_{g}$, we construct a local, diffeomorphism invariant trace on the Kauffman bracket skein module $K_{t}\left(\Sigma_{g} \times I\right)$. The trace is defined when $|t|$ is neither 0 nor 1 , and at certain roots of unity. At $t=-1$, the trace is integration against the symplectic measure on the $S U(2)$ character variety of the fundamental group of $\Sigma_{g}$.
\end{abstract}

Mathematics Subject Classification (2000). 57M27.

Keywords. Skein, quantum invariant, Kauffman bracket, trace, symplectic measure.

\section{Introduction}

The Kauffman bracket skein module of a 3-manifold is a complex vector space with isotopy classes of knots in the manifold as its basis, divided by the relation corresponding to the Kauffman bracket link invariant. This relation involves a complex parameter, $t$. We define the Yang-Mills measure in the Kauffman bracket skein algebra of a cylinder over a closed surface $\Sigma_{g}$ in an elementary way, and show that it is a diffeomorphism-invariant trace. From a diagrammatic viewpoint, the Yang-Mills measure is the extension of the Kauffman bracket to framed link diagrams lying in any compact oriented surface.

The proof of its convergence for $|t| \neq 1$ uses estimates involving quantum $6 j$ symbols. When the deformation parameter is a generic point on the unit circle, then the measure does not converge. At roots of unity it coincides with Turaev's shadow world invariant [25], which is shown in a subsequent paper [10]. Finally, at $\mathrm{t}=-1$, the Yang-Mills measure is integration against the symplectic measure on the space $\mathcal{M}\left(\Sigma_{g}\right)$ of conjugacy classes of representations of the fundamental group of $\Sigma_{g}$ into $S U(2)$.

Since the introduction of quantum invariants of 3 -manifolds [21, 27] the fact that they are only defined at roots of unity has been an obstruction to analyzing their properties. One approach has been to study the perturbative theory of quan-

This research was partially supported by NSF-DMS-9803233 and NSF-DMS-9971905. 
tum invariants [18]. However, there is ample evidence quantum invariants of three manifolds exist as holomorphic functions on the unit disk, that diverge everywhere on the unit circle but at roots of unity [15]. This paper takes a step towards seeing that this holds in general. The Yang-Mills measure is the path integral on a topological quantization [3] of the $S U(2)$-characters of the fundamental group of a closed surface. The measure displays the same convergence properties as are expected of quantum invariants of 3-manifolds.

The definition of the symplectic structure and formulas for its computation are in $[11,12]$. The volume of $\mathcal{M}\left(\Sigma_{g}\right)$ was computed by Witten in [28] in two ways: via the equivalence of two computations in quantum field theory, and by noting that the symplectic measure is equal to the measure coming from Reidemeister torsion. In Witten's setting the Yang-Mills measure is a path integral in a lattice model of field theory that depends on area. Forman [7] gave a direct proof that Witten's measure converges to the symplectic measure as the area goes to zero.

The construction in this paper is motivated by our work extending ideas of $[1,6]$. Alekseev, Grosse and Schomerus [1] conceived of a method of constructing lattice gauge field theory based on a quantum group. This idea was further developed by Buffenoir and Roche [6] who gave a construction of the algebra, its Wilson loops and a trace called the Yang-Mills measure that were completely analogous to Witten's construction. Their theory is topological when the area is set to zero. We recognized that the algebra of observables from their theories could be understood as the Kauffman bracket skein module of a cylinder over a surface $[4,5]$. These considerations lead one to expect that the Yang-Mills measure exists as a trace on the Kauffman bracket skein algebra of a closed surface. In this paper we affirm this fact.

This paper is organized as follows. Section 2 recalls definitions, associated formulas and the algebra structure of the Kauffman bracket skein module of a cylinder over a surface. In Section 3 the Yang-Mills measure is defined for compact surfaces with boundary, and is proved to be a trace. In Section 4, working with the parameter $t$ such that $|t| \neq 1$, we obtain estimates for the absolute value of the tetrahedral coefficients and use these to show that the Yang-Mills measure can be defined for closed surfaces. In Section 5 we define and investigate the measure when $t$ is a root of unity.

\section{Preliminaries}

Let $M$ be an orientable 3-manifold. A framed link in $M$ is an embedding of a disjoint union of annuli into $M$. Framed links are depicted by showing the core of an annulus lying parallel to the plane of the paper (i.e. with blackboard framing). Two framed links in $M$ are equivalent if there is an isotopy of $M$ taking one to the other. Let $\mathcal{L}$ denote the set of equivalence classes of framed links in $M$, including the empty link. Fix a complex number $t \neq 0$. Consider the vector space $\mathbb{C} \mathcal{L}$ with 
basis $\mathcal{L}$. Define $S(M)$ to be the smallest subspace of $\mathbb{C} \mathcal{L}$ containing all expressions of the form

$$
\left.\grave{\prime}-t \longleftarrow-t^{-1}\right)(
$$

and

$$
\bigcirc \cup L+\left(t^{2}+t^{-2}\right) L
$$

where the framed links in each expression are identical outside balls pictured in the diagrams. The Kauffman bracket skein module $K_{t}(M)$ is the quotient

$$
\mathbb{C} \mathcal{L} / S(M) \text {. }
$$

Elements of $K_{t}(M)$ are called skeins. Let $F$ be a compact orientable surface and let $I=[0,1]$. There is an algebra structure on $K_{t}(F \times I)$ that comes from laying one framed link over the other. Suppose that $\alpha, \beta \in K_{t}(F \times I)$ are skeins represented by framed links $L_{\alpha}$ and $L_{\beta}$. After isotopic deformations, to "lower" the first framed link and "raise" the second, $L_{\alpha} \subset F \times\left[0, \frac{1}{2}\right)$ and $L_{\beta} \subset F \times\left(\frac{1}{2}, 1\right]$. The skein $\alpha * \beta$ is represented by $L_{\alpha} \cup L_{\beta}$. This product extends to a bilinear product on $K_{t}(F \times I)$. We denote the resulting algebra by $K_{t}(F)$ to emphasize that it comes from viewing the underlying three manifold as a cylinder over $F$.

The notation and the formulas in this paper are taken from [14]. However, the variable $t$ replaces $A$, and we use quantum integers

$$
[n]=\frac{t^{2 n}-t^{-2 n}}{t^{2}-t^{-2}}
$$

When $t= \pm 1,[n]=n$. Note that $\Delta_{n}$ from [14] is equal to $(-1)^{n}[n+1]$.

There is a standard convention for using a framed trivalent graph $\Gamma \subset M$ to model a skein. When $\Gamma$ is represented by a diagram we assume blackboard framing. An admissible coloring of $\Gamma$ is an assignment of a nonnegative integer to each edge so that the colors at trivalent vertices form admissible triples (defined below). The corresponding skein in $K_{t}(M)$ is obtained by inserting the $m$-th Jones-Wenzl idempotent into each edge labeled with the letter $m$, inserting Kauffman triads at the vertices, and joining up with parallel strands. Jones-Wenzl idempotents (see [26], or [16], p.136) can be defined recursively as in Figure 1, where the coefficient $c$ is equal to $\frac{[n-1]}{[n]}$. A Kauffman triad (see [16, Fig. 14.7]) is pictured in Figure 2.

Recall the fusion identity:

$$
\left.\right|^{a}=\sum_{c}(-1)^{c} \frac{[c+1]}{\theta(a, b, c)} \sum_{c}^{a}{ }_{b}^{b}
$$

where the sum is over all $c$ so that the triples $(a, b, c)$ are admissible, i.e. $a+b+c$ is even, $a \leq b+c, b \leq a+c$, and $c \leq a+b$. The value of $\theta(a, b, c)$ is given by equation (14) below. The fusion relation is satisfied in $K_{t}(M)$ unless $t$ is a root of unity other than \pm 1 . 


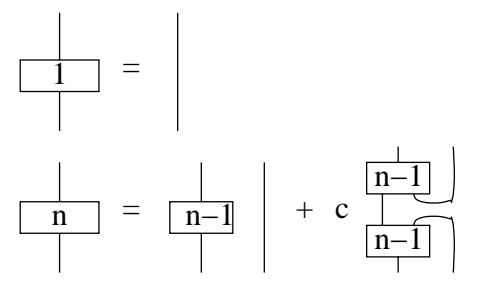

Figure 1. Jones-Wenzl idempotents

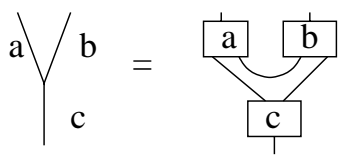

Figure 2. Kauffman triad

\section{The Yang-Mills measure in a handlebody}

Throughout this section we assume that $t$ is not a root of unity. The first result is well known and comes from Przytycki's [19] construction of examples of torsion in skein modules.

Lemma 1 (The Sphere Lemma). Let $s_{c}$ be a skein represented by coloring a trivalent framed graph in the manifold $M$. Suppose further that there is a sphere embedded in $M$ which intersects the underlying graph transversely in a single point in the interior of an edge, and the color of that edge is not zero. Then $s_{c}=0$.

Proof. Using the "light bulb trick" isotope the framed graph $s_{c}$ so that it is the same graph, but the framing on the edge intersecting the sphere has been changed by adding two kinks. Using the formula for eliminating a kink, notice that $s_{c}$ is a nontrivial complex multiple of itself. Ergo, $s_{c}$ represents zero in $K_{t}(M)$.

Consider now $K_{t}\left(\#{ }_{g} S^{1} \times S^{2}\right)$, the Kauffman bracket skein module of the connected sum of $g$ copies of $S^{1} \times S^{2}$.

Proposition 1. The skein module $K_{t}\left(\#{ }_{g} S^{1} \times S^{2}\right)$ is canonically isomorphic to $\mathbb{C}$. The isomorphism is given by writing each skein as a complex multiple of the empty skein.

Proof. This follows easily from theorems of Hoste and Przytycki $[13,19,20]$. In [13] the Kauffman bracket skein module of $S^{1} \times S^{2}$ is computed over $\mathbb{Z}\left[t, t^{-1}\right]$. This along with the results in [20] on the Kauffman bracket skein module of a connected 
sum over rational functions in $t$, combined with the universal coefficient theorem stated in [19], proves the desired result.

We outline the actual isomorphism with the complex numbers. Choose a system of spheres in $\#{ }_{g} S^{1} \times S^{2}$ that cut it down to a punctured ball. Given a skein in $\#_{g} S^{1} \times S^{2}$, represent it as a linear combination of colored, framed, trivalent graphs intersecting the spheres transversely in interior of edges, and so that each graph intersects any sphere at most once. This is done by fusing multiple edges passing through the same sphere. By the sphere lemma, we can assume the graphs miss the spheres. Now take the Kauffman bracket of the skein in the punctured ball to write it as a complex multiple of the empty skein.

Given a handlebody $H$ of genus $g$, its double is $\#_{g} S^{1} \times S^{2}$. There is a linear functional $\mathcal{Y} \mathcal{M}: K_{t}(H) \rightarrow \mathbb{C}$ computed by taking the inclusion of $H$ into $\#_{g} S^{1} \times$ $S^{2}$ followed by taking the "Kauffman bracket" as above. Let $F$ be a compact, oriented surface with boundary. Since $F \times I$ is a handlebody the linear functional

$$
\mathcal{Y} \mathcal{M}: K_{t}(F) \rightarrow \mathbb{C}
$$

is defined. We call this the Yang-Mills measure.

Choose a trivalent spine of $F$. Skeins given by the admissible colorings of that spine form a basis for $K_{t}(F)$. The skein modules of the disk and annulus are exceptions; the first is spanned by the empty skein and the latter is described in Section 4. In terms of this basis the Yang-Mills measure is just the coefficient of the skein coming from labeling all the edges of the spine with 0 .

Proposition 2. The Yang-Mills measure is a trace, that is

$$
\mathcal{Y} \mathcal{M}(\alpha * \beta)=\mathcal{Y} \mathcal{M}(\beta * \alpha) \text {. }
$$

Furthermore, the trace is invariant under the action of the diffeomorphisms of $F \times I$ on $K_{t}(F)$.

Proof. Let $L$ be the link $\partial F \times\{1 / 2\}$. The result of removing $L$ from the double of $F \times I$ is homeomorphic to the Cartesian product of the interior of $F$ with a circle. Given any skein in $F \times I$ we can represent it by a linear combination of framed links that miss $L$. Hence, the Yang-Mills measure factors through the skein module of $F \times S^{1}$. In $F \times S^{1}$ the skeins $\alpha * \beta$ and $\beta * \alpha$ are the same.

The group of diffeomorphisms of the handlebody $F \times I$ acts on $K_{t}(F)$ in the obvious way. If $f: F \times I \rightarrow F \times I$ is a diffeomorphism then it can be extended to $D f: \#_{g} S^{1} \times S^{2} \rightarrow \# S_{g} S^{1} \times S^{2}$. Since the image of the empty skein under a diffeomorphism is the empty skein, the action of $D f$ on $K_{t}\left(\# H_{g} S^{1} \times S^{2}\right)$ is trivial. Therefore, $\mathcal{Y} \mathcal{M}(f(\alpha))=\mathcal{Y} \mathcal{M}(\alpha)$.

The final commonly used property of the Yang-Mills measure is that it is local. Suppose that $k$ is a proper arc in $F$. Cut $F$ along $k$ to get a surface $F^{\prime}$. If we write a skein $\alpha$ as a linear combination of skeins given by admissibly colored graphs, 
each one intersecting $k$ transversely in at most a single point, in evaluating the Yang-Mills measure of that skein we can throw out any graph such that the edge intersecting $k$ carries a nonzero label. This yields a skein in $F^{\prime}$, denoted by $\alpha_{k}$. Then $\mathcal{Y} \mathcal{M}(\alpha)=\mathcal{Y} \mathcal{M}\left(\alpha_{k}\right)$.

\section{The Yang-Mills measure on a closed surface}

Throughout this section assume that $|t| \neq 1$. In fact, we only work with $0<t<1$. However, the same proofs are valid when $1<t$ since the formulas are symmetric in $t$ and $t^{-1}$. Finally, the arguments extend to the case where $t$ is not real by replacing the estimates for $t \in \mathbb{R}$ by estimates of the absolute value of $t \in \mathbb{C}$.

Recall the Kauffman bracket skein algebra of a cylinder over an annulus $A$. The central core of the annulus can be seen as a link by giving it the blackboard framing. Let $s_{i}$ be the skein in the annulus which is the result of coloring the core with the $i$-th Jones-Wenzl idempotent. The skein module $K_{t}(A)$ is the vector space with basis $\left\{s_{i}\right\}$, where $i$ runs from zero to infinity. The product with respect to this basis is given by

$$
s_{i} * s_{j}=\sum_{q \in I_{i, j}} s_{q},
$$

where $I_{i, j}=\{q|| i-j \mid \leq q \leq i+j, q \equiv i+j \bmod 2\}$.

Use the Yang-Mills measure on $K_{t}(A)$ to define a pairing:

$$
\langle\alpha, \beta\rangle=\mathcal{Y} \mathcal{M}(\alpha * \beta) \text {. }
$$

The $s_{i}$ form an orthonormal basis with respect to $(9)$. This pairing identifies the linear dual of $K_{t}(A)$ with series of the form $\sum_{i} \alpha_{i} s_{i}$, where the $\alpha_{i}$ are complex numbers. Note that:

$$
\left\langle\sum_{i=0}^{\infty} \alpha_{i} s_{i}, \sum_{j=0}^{n} \beta_{j} s_{j}\right\rangle=\sum_{i=0}^{n} \alpha_{i} \beta_{i} .
$$

Let $\Sigma_{g, 1}$ denote the compact orientable surface of genus $g$ with one boundary component. There is a pairing,

$$
K_{t}(A) \otimes K_{t}\left(\Sigma_{g, 1}\right) \rightarrow K_{t}\left(\Sigma_{g, 1}\right)
$$

given by representing the skein in $K_{t}\left(\Sigma_{g, 1}\right)$ by a linear combination of links disjoint from some collar of the boundary, and plugging the skein in $K_{t}(A)$ into the collar. The Yang-Mills measure can then be applied to give a pairing,

$$
K_{t}(A) \otimes K_{t}\left(\Sigma_{g, 1}\right) \rightarrow \mathbb{C}
$$

by letting $\langle\alpha, \sigma\rangle=\mathcal{Y} \mathcal{M}(\alpha * \sigma)$. Topologize $K_{t}(A)$ by giving it the weak topology from this pairing. That is, a sequence $\sigma_{n} \in K_{t}(A)$ is Cauchy if for every skein $\alpha \in K_{t}\left(\Sigma_{g, 1}\right)$, the sequence of complex numbers $\mathcal{Y} \mathcal{M}\left(\alpha * \sigma_{n}\right)$ is Cauchy. Embed $K_{t}(A)$ into the equivalence classes of Cauchy sequences in the standard way and 
take its closure to form a completion. A linear functional on $K_{t}\left(\Sigma_{g, 1}\right)$ that comes from an element of this completion via the pairing (12) is called a distribution. It is interesting to note that the weak topology on $K_{t}(A)$ depends on the genus of the surface.

There is a map of $K_{t}\left(\Sigma_{g, 1}\right)$ into $K_{t}\left(\Sigma_{g}\right)$ induced by inclusion. The kernel of this map is spanned by handle-slides. A handle-slide is a skein that is represented by the difference of two links such that one can be obtained from the other by a slide across an imagined disk filling the boundary of $\Sigma_{g, 1}$. If $g>1$ there is a distribution on $K_{t}\left(\Sigma_{g, 1}\right)$ which annihilates all handle-slides. This linear functional descends to the skein module of the closed surface. The Yang-Mills measure on a closed surface is the result of evaluating this distribution followed by a normalization.

Let's think about what a skein in $K_{t}(A)$ would be like if it annihilated all handle-slides. Begin by writing it as $\sum_{i} \alpha_{i} s_{i}$ and solve for the $\alpha_{i}$. A simple computation shows that if $\alpha_{0}$ is zero then all $\alpha_{i}$ are zero. Normalize so that $\alpha_{0}=1$. Notice that if our skein annihilates handle-slides then the skein $s_{1}+[2] s_{0}$ must be annihilated. Using the rules for multiplication (8) we see that the coefficient $\alpha_{1}$ is equal to $-[2]$. Continuing on this way we see that this skein has to be

$$
\sum_{i=0}^{\infty}(-1)^{i}[i+1] s_{i}
$$

which is of course not in $K_{t}(A)$.

The first goal is to show that for $g>1$ the sequence of partials sums $\sum_{i=0}^{n}(-1)^{i}[i+$ $1] s_{i}$ is Cauchy in the weak topology from $Y$, and so defines a distribution.
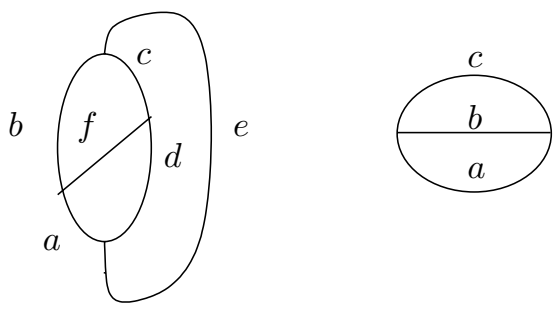

Figure 3. Tet and theta

The notation Tet $\left(\begin{array}{lll}a & b & e \\ c & d & f\end{array}\right)$ stands for the value of the skein pictured in Figure 3 on the left, in the skein space of a ball, identified with the complex numbers. The explicit formula is given in [14]. We also need the quantity $\theta(a, b, c)$ which is the value of the skein modeled on the colored graph on the right in Figure 3. In terms of quantum integers

$$
\theta(a, b, c)=(-1)^{\frac{a+b+c}{2}} \frac{\left[\frac{a+b+c}{2}+1\right] !\left[\frac{a+b-c}{2}\right] !\left[\frac{b+c-a}{2}\right] !\left[\frac{c+a-b}{2}\right] !}{[a] ![b] ![c] !} .
$$


Another quantity, called a $6 j$ symbol, is derived from the tetrahedral evaluation. Specifically,

$$
\left\{\begin{array}{lll}
a & b & e \\
c & d & f
\end{array}\right\}=\frac{\operatorname{Tet}\left(\begin{array}{lll}
a & b & e \\
c & d & f
\end{array}\right)(-1)^{e}[e+1]}{\theta(a, d, e) \theta(c, b, e)} .
$$

The $6 j$ symbols are the coefficients of the change of basis matrix for recoupling on graphs [14]. As a consequence they satisfy an orthogonality equation:

$$
\sum_{e}\left\{\begin{array}{lll}
a & b & e \\
c & d & f
\end{array}\right\}\left\{\begin{array}{lll}
d & a & g \\
b & c & e
\end{array}\right\}=\delta_{f}^{g}
$$

where $\delta_{f}^{g}$ is the Kronecker delta, and the sum is over all $e$ such that triples $(b, c, e)$, $(a, d, e)$ are admissible.

The following proposition seems quite weak, but turns out to be a powerful tool for gauging the convergence of series of Kauffman brackets.

\section{Proposition 3.}

$$
\left|\operatorname{Tet}\left(\begin{array}{lll}
a & b & e \\
c & d & f
\end{array}\right)\right| \leq \sqrt{\frac{\theta(b, c, e) \theta(a, d, e) \theta(a, b, f) \theta(c, d, f)}{(-1)^{e+f}[e+1][f+1]}}
$$

Proof. In order for all the triples at the vertices of a tetrahedron to be admissible, the parity of the sum of the entries in any two columns of

$$
\operatorname{Tet}\left(\begin{array}{lll}
a & b & e \\
c & d & f
\end{array}\right)
$$

has to be the same. Use (15) to expand the formulas for the $6 j$ symbols in the orthogonality relation (16), with $g=f$. The tetrahedral evaluations are equal and the signs of the $\theta$ 's and the $(-1)^{e+f}$ cancel so that each term in the sum is positive. Hence every term in the sum is less than 1. Fixing $e$ and putting everything except for the tetrahedral evaluations on the right hand side, and taking square roots yields the desired result.

Corollary 1. There is a real valued function $C\left(k_{1}, k_{2}, k_{3}\right)$ so that for all $t, 0<$ $t<1$

$$
\frac{\left|\operatorname{Tet}\left(\begin{array}{ccc}
i & i & i \\
k_{1} & k_{2} & k_{3}
\end{array}\right)\right|}{\sqrt{\left|\theta\left(i, i, k_{1}\right) \theta\left(i, i, k_{2}\right) \theta\left(i, i, k_{3}\right)\right|}}
$$

is less than $t^{i} C\left(k_{1}, k_{2}, k_{3}\right)$ whenever the graphs corresponding to the functions in the formula are admissibly labeled. 
Proof. Substitute into inequality (17) from Proposition 3 to get,

$$
\left|\operatorname{Tet}\left(\begin{array}{ccc}
i & i & i \\
k_{1} & k_{2} & k_{3}
\end{array}\right)\right| \leq \sqrt{\frac{\theta\left(k_{1}, k_{2}, k_{3}\right) \theta\left(i, i, k_{1}\right) \theta\left(i, i, k_{2}\right) \theta\left(i, i, k_{3}\right)}{(-1)^{i+k_{3}}\left[k_{3}+1\right][i+1]}} .
$$

Shift $\sqrt{\left|\theta\left(i, i, k_{1}\right) \theta\left(i, i, k_{2}\right) \theta\left(i, i, k_{3}\right)\right|}$ to the left hand side. Use the fact that $\frac{1}{[i+1]} \leq$ $t^{2 i}$ to make the right hand side bigger. Finally, note that the remaining factor on the right hand side is a function of $k_{1}, k_{2}$ and $k_{3}$.

Theorem 1. The sequence $\sum_{i=0}^{n}(-1)^{i}[i+1] s_{i}$ defines a distribution for $g>1$. That is, the limit

$$
\mathcal{Y} \mathcal{M}_{D}(\alpha)=\lim _{n \rightarrow \infty} \mathcal{Y M}\left(\alpha * \sum_{i=0}^{n}(-1)^{i}[i+1] s_{i}\right)
$$

exists and gives a well defined trace on $K_{t}\left(\Sigma_{g, 1}\right)$ when $g>1$.

Proof. Choose a trivalent spine for $\Sigma_{g, 1}$ with $4 g-2$ vertices and $6 g-3$ edges. Basis elements $s_{c}$ for $K_{t}\left(\Sigma_{g, 1}\right)$ correspond to labeling the edges admissibly with integers $k_{j}$, where $j$ runs from 1 to $6 g-3$. Let $A$ denote an annulus which is a collar of the boundary of $\Sigma_{g, 1}$. Let $s_{i}$ denote the core of $A$ labeled with the $i$-th JonesWenzl idempotent. In order to compute $\mathcal{Y} \mathcal{M}\left(s_{c} * s_{i}\right)$ place both skeins in the same diagram. Choose a system of arcs, each intersecting this configuration transversely in three points, that isolate the vertices from one another. The transverse points of intersection are labeled $i, k_{j}, i$ as you traverse each arc. Fuse along these arcs, until the resulting graphs intersect each arc in at most one point. Discard any term where the label on an edge intersecting an arc is not zero. Given a vertex $v$, let $\left(k_{v 1}, k_{v 2}, k_{v 3}\right)$ be the triple of colors appearing there. The resulting answer is:

$$
\mathcal{Y} \mathcal{M}\left(s_{c} * s_{i}\right)=\prod_{j=1}^{6 g-3} \frac{1}{\theta\left(i, i, k_{j}\right)} \prod_{v} \operatorname{Tet}\left(\begin{array}{ccc}
i & i & i \\
k_{v 1} & k_{v 2} & k_{v 3}
\end{array}\right) \text {. }
$$

Each edge appears at exactly two vertices, so (22) can be written as a product of $4 g-2$ factors like (19). By Corollary 1 the absolute value of $\mathcal{Y} \mathcal{M}\left(s_{c} * s_{i}\right)$ is less than $C\left(k_{j}\right) t^{i(4 g-2)}$, where $C\left(k_{j}\right)$ is a number depending only on the $k_{j}$. The $n$-th partial sum for $\mathcal{Y} \mathcal{M}_{D}\left(s_{c}\right)$ is

$$
\sum_{i=0}^{n}(-1)^{i}[i+1] \prod_{j=1}^{6 g-3} \frac{1}{\theta\left(i, i, k_{j}\right)} \prod_{v} \operatorname{Tet}\left(\begin{array}{ccc}
i & i & i \\
k_{v 1} & k_{v 2} & k_{v 3}
\end{array}\right) .
$$

Note that $[i+1]$ is less than $(i+1) t^{-2 i}$. Hence the $i$-th summand is less than $(i+1)(-1)^{i} C\left(k_{j}\right) t^{i(4 g-4)}$. The ratio test implies that the sequence of partial sums is absolutely convergent for $0<t<1$.

Finally, $\mathcal{Y M}_{\mathcal{D}}$ is a trace since the partial sums $\sum_{i=0}^{n}(-1)^{i}[i+1] s_{i}$ can be seen as lying in the center of $K_{t}\left(\Sigma_{g, 1}\right)$. 


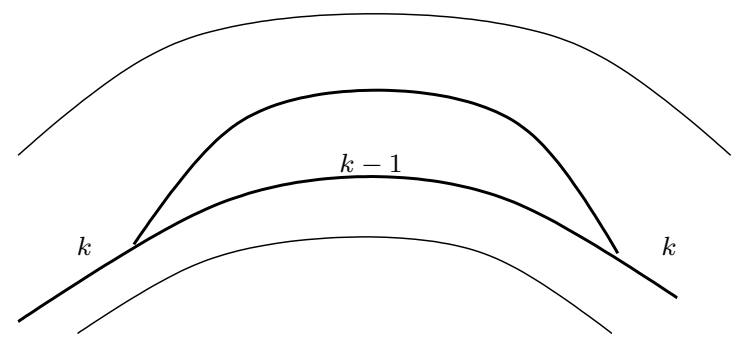

Figure 4

Theorem 2. $\mathcal{Y} \mathcal{M}_{D}$ descends to give a well defined trace

$$
\mathcal{Y} \mathcal{M}: K_{t}\left(\Sigma_{g}\right) \rightarrow \mathbb{C} .
$$

Proof. There is a homomorphism $K_{t}\left(\Sigma_{g, 1}\right) \rightarrow K_{t}\left(\Sigma_{g}\right)$ induced by inclusion, since the surface $\Sigma_{g}$ is the result of adding a disk to the boundary of surface $\Sigma_{g, 1}$. The kernel of this homomorphism consists of all skeins that can be written as a linear combination of handle-slides [19]. The next step is to show that the linear functional $\mathcal{Y} \mathcal{M}_{D}$ annihilates all handle-slides. To this end we analyze the difference of the two skeins in the annulus (relative to a pair of points in the boundary).

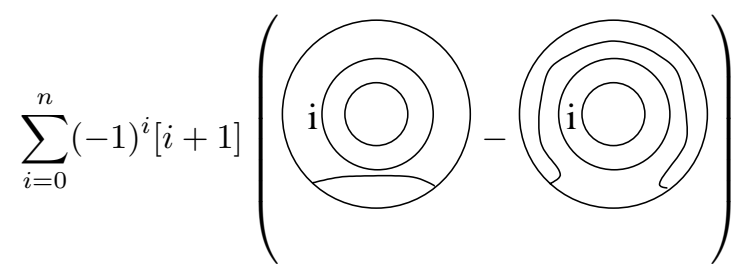

The analysis of the diagram (25) is due to Lickorish, [16]. It is equal to:

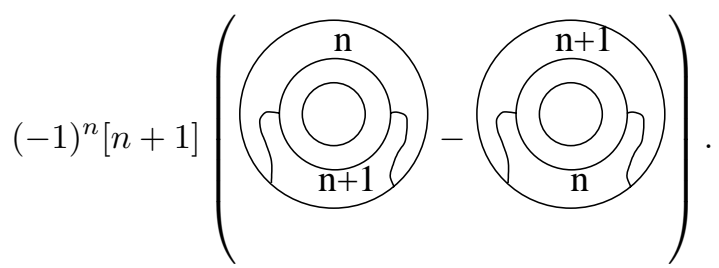

This diagram needs to be set in place. Using standard arguments as in [2] yields that we only need to check handle-slides of the following form. Take a skein corresponding to a colored spine, and separate one strand along an edge as in Figure 4.

Now slide the strand over the added disk, locally the diagram looks like Figure 5 .

Multiplying each of the diagrams in Figures 4 and 5 by $\sum_{i=0}^{n}(-1)^{i}[i+1] s_{i}$, taking their difference, and using the identity $(25)=(26)$, we get a difference of 


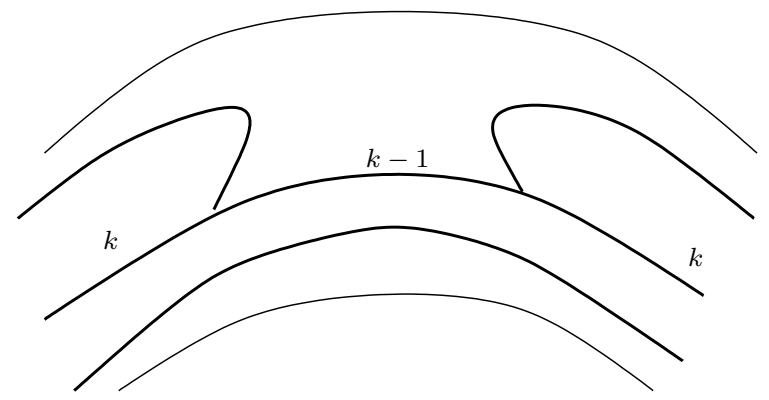

Figure 5

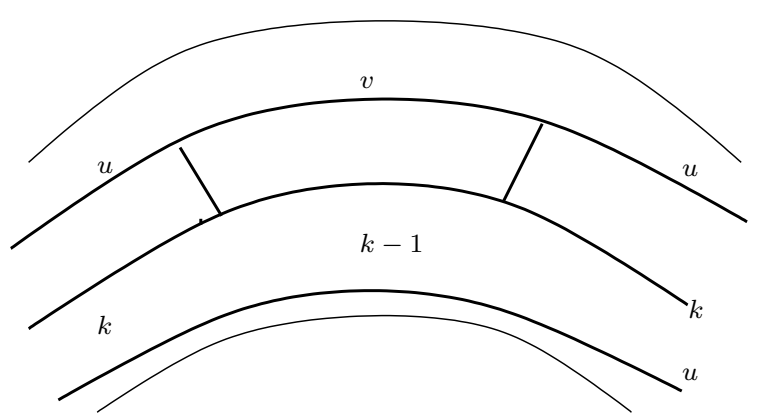

Figure 6

two terms like the one in Figure 6 . In the first one the label $u=n$ and the label $v=n+1$, and in the second one $u=n+1$ and $v=n$.

Fusing to isolate the vertices of this diagram requires two more cross cuts than the diagrams we have been working with up till now. We get the product of

$$
(-1)^{n}[n+1] \frac{1}{\theta(u, k, u) \theta(u, k-1, v)} \operatorname{Tet}\left(\begin{array}{lll}
u & u & v \\
1 & k-1 & k
\end{array}\right) \operatorname{Tet}\left(\begin{array}{llc}
u & v & u \\
1 & k & k-1
\end{array}\right)
$$

with the standard product,

$$
\prod_{j=1}^{6 g-3} \frac{1}{\theta\left(u, u, k_{j}\right)} \prod_{v} \operatorname{Tet}\left(\begin{array}{ccc}
u & u & u \\
k_{v 1} & k_{v 2} & k_{v 3}
\end{array}\right) .
$$

The product $(28)$ is smaller than a global constant, depending on the $k_{j}$, times $t^{n(4 g-2)}$. It remains to ascertain that the term $(27)$ is not too large. Direct application of the inequality from Proposition 3, together with the fact that $\theta(a+$ $1, a, 1)=[a+2]$, yields that ( regardless of whether $u=n$ and $v=n+1$, or $u=n+1$ and $v=n)$ the absolute value of $(27)$ is less than $\frac{[n+1][n+2][k+1]}{\sqrt{[n+1][n+2][k][k+1]}}$ which is less than a constant depending on $k$ times $t^{-2 n}$.

As long as the genus of the surface is greater than 1, the full product goes to 
zero as $n$ goes to infinity. So, in the limit, all handle-slides are annihilated.

The case of a surface of genus 1 is slightly different. To get a nontrivial convergent distribution we need to divide the partial sum $\sum_{i=0}^{n}(-1)^{i}[i+1] s_{i}$ by $n$. The sequence is then Cauchy and defines a distribution on $K_{t}\left(T^{2}\right)$. That is, the limit

$$
\mathcal{Y} \mathcal{M}_{D}(\alpha)=\lim _{n \rightarrow \infty} \mathcal{Y} \mathcal{M}\left(\alpha * \frac{1}{n} \sum_{i=0}^{n}(-1)^{i}[i+1] s_{i}\right)
$$

exists and gives a well defined trace on the algebra of a torus with one boundary component. This trace descends to a well defined trace on $K_{t}\left(T^{2}\right)$.

The algebra $K_{t}\left(T^{2}\right)$ is very nice for working examples. If $(p, q)$ is a pair of integers that are relatively prime there is an obvious skein $s_{(p, q)}$ in $K_{t}\left(T^{2}\right)$ corresponding to the $(p, q)$ curve on the torus. Define a family of skeins based on $(p, q)$ by using the following iterative scheme: $s_{(p, q)_{0}}=2 s_{(0,0)}$, that is, twice the empty skein, and $s_{(p, q)_{1}}=s_{(p, q)}$. For $d>1$ define:

$$
s_{(p, q)_{d}}=s_{(p, q)} * s_{(p, q)_{d-1}}-s_{(p, q)_{d-2}} .
$$

Finally, if $d=\operatorname{gcd}\{p, q\}$, let

$$
c_{(p, q)}=s_{(p / d, q / d)_{d}} .
$$

In the special case of $(0,0)$ let $c_{(0,0)}=2 s_{(0,0)}$ Using this notation the product in $K_{t}\left(T^{2}\right)$ is given by

$$
c_{(p, q)} * c_{(u, v)}=t\left|\begin{array}{ll}
p & q \\
u & v
\end{array}\right|_{c_{(p+u, q+v)}+t}-\left|\begin{array}{ll}
p & q \\
u & v
\end{array}\right|_{c_{(p-u, q-v)}}
$$

The formula (32) is proven in [8].

There is a map

$$
\mu: K_{t}\left(T^{2}\right) \rightarrow \mathbb{C} \emptyset \oplus \mathbb{C} H_{1}\left(T^{2} ; Z_{2}\right)
$$

introduced in [17]. Let

$$
\mu\left(\sum_{(p, q)} a_{(p, q)} c_{(p, q)}\right)=a_{(0,0)} \emptyset+\sum_{(p, q) \neq(0,0)} a_{(p, q)}[(p, q)],
$$

where $[(p, q)]$ is the $Z_{2}$-homology class in $H_{1}\left(T^{2} ; Z_{2}\right)$ corresponding to $d=\operatorname{gcd}\{p, q\}$ copies of a $(p / d, q / d)$ curve on the torus. The map $\mu$ has as its kernel the submodule of all commutators. Hence any linear functional on the five dimensional space that is the image of $\mu$ is a trace. It is easy to check that there is a three dimensional family of traces that are invariant under diffeomorphism. In this set up

$$
\mathcal{Y} \mathcal{M}\left(\sum_{(p, q)} a_{(p, q)} c_{(p, q)}\right)=a_{(0,0)}
$$


This is the same trace as the one induced from the inclusion of $K_{t}\left(T^{2}\right)$ into the non-commutative torus [8].

Towards uniqueness of the Yang-Mills measure, it should be normalized, just as the symplectic measure on moduli space needs to be normalized. It should also be invariant under diffeomorphism, and be local. Locality is made up by two rules. One for removing a point from a closed surface, and one for cutting a surface with boundary along an arc. To be curt, locality for a closed surface $\Sigma_{g}$ is the statement that the Yang-Mills measure is derived from the Yang-Mills measure on a surface with boundary, as we have done in this paper. That is, there is a surjection $K_{t}\left(\Sigma_{g, 1}\right) \rightarrow K_{t}\left(\Sigma_{g}\right)$ where $\Sigma_{g, 1}$ is the result of removing an open disk from $\Sigma_{g}$. The Yang-Mills measure on $K_{t}\left(\Sigma_{g}\right)$ pulls back to $\mathcal{Y} \mathcal{M}_{D}: K_{t}\left(\Sigma_{g, 1}\right) \rightarrow \mathbb{C}$. If $g>1$, the value of the map $\mathcal{Y M}_{D}$ on any $\alpha$ is $\mathcal{Y} \mathcal{M}\left(\alpha * \sum_{i}(-1)^{i}[i+1] s_{i}\right)$. If $g=1$, the map $\mathcal{Y} \mathcal{M}_{D}$ is defined as in (29). The map $\mathcal{Y} \mathcal{M}_{D}$ could be characterized more abstractly than this, but it would lead to analytic complications that are not appropriate for this exposition. When the surface $\Sigma$ has boundary and $\kappa$ is a properly embedded arc in $\Sigma$, let $\Sigma^{\prime}$ be the result of cutting $\Sigma$ along $\kappa$. Let $N_{t}(\Sigma)$ be the linear subspace of $K_{t}(\Sigma)$ spanned by skeins that are represented by admissibly colored trivalent graphs that intersect $\kappa$ in a single point of transverse intersection, so that the edge intersecting $\kappa$ carries a nonzero label. Let $\iota: K_{t}\left(\Sigma^{\prime}\right) \rightarrow K_{t}(\Sigma)$ be the natural inclusion map. Then

$$
K_{t}(\Sigma)=\operatorname{im}(\iota) \oplus N_{t}(\Sigma)
$$

Locality is the statement that $\mathcal{Y} \mathcal{M}$ is zero on $N_{t}(\Sigma)$ and its restriction to $\operatorname{im}(\iota)$ pulls back to $\mathcal{Y} \mathcal{M}$ on $K_{t}\left(\Sigma^{\prime}\right)$.

Theorem 3. Let $\Sigma$ be a compact surface. The Yang-Mills measure is the unique, local, diffeomorphism invariant trace on $K_{t}(\Sigma)$ such that

- $\mathcal{Y M}(\emptyset)=1$ when $\Sigma$ has boundary and when $\Sigma$ is a torus;

- $\mathcal{Y M}(\emptyset)=\sum_{i=0}^{\infty} \frac{1}{[i+1]^{(2 g-2)}}$ when $\Sigma$ is a closed surface of genus $g>1$.

Proof. Since $K_{t}\left(D^{2}\right)=\mathbb{C}$, up to normalization there is only one trace. For more complicated surfaces with boundary we argue by induction on the Euler characteristic, using locality for surfaces with boundary for the inductive step. Uniqueness for closed surfaces follows from the other form of locality. This trace is diffeomorphism invariant by Proposition 2 .

\section{Roots of unity}

Fusion no longer holds in $K_{t}(M)$ when $t$ is a root of unity. However, when $t=e^{\frac{\pi i}{2 r}}$ then one can take a quotient, where an appropriate form of the fusion identity is true. This can be done be taking the quotient of $K_{t}(M)$ by the submodule spanned by skeins corresponding to trivalent graphs where some edge carries the 
label $r-1$. The quotient is denoted $K_{r, f}(M)$. The reduced skein $K_{r, f}(M)$ is a central object in the construction of quantum invariants of 3 -manifolds [9, 22, 23].

The Yang-Mills measure on a surface with boundary is obtained the same way as for other values of $t$. Since $[r]=0$, the iterative procedure for finding a skein in the annulus that annihilates handle-slides terminates, to yield

$$
\sum_{i=0}^{r-2}(-1)^{i}[i+1] s_{i} \text {. }
$$

There is an induced trace,

$$
\mathcal{Y} \mathcal{M}: K_{r, f}\left(\Sigma_{g}\right) \rightarrow \mathbb{C}
$$

constructed the same way as for other $t$ except that there is no need to take a limit because the formula is a finite sum.

Notice that $\Sigma_{g}$ is the boundary of some handlebody $H_{g}$. There is an action of $K_{r, f}\left(\Sigma_{g}\right)$ on $K_{r, f}\left(H_{g}\right)$ given by gluing skeins in $\Sigma_{g} \times I$ into a collar of the boundary of $H_{g}$. The action gives a map

$$
\phi: K_{r, f}\left(\Sigma_{g}\right) \rightarrow \operatorname{End}\left(K_{r, f}\left(H_{g}\right)\right) .
$$

As we are working at a root of unity, $K_{r, f}\left(H_{g}\right)$ is a finite dimensional vector space. Denote its dimension by $d$ and let $\omega=\mathcal{Y} \mathcal{M}(\emptyset)=\sum_{i=0}^{r-2} \frac{1}{[i+1]^{2 g-2}}$.

Proposition 4. The Yang-Mills measure $\mathcal{Y} \mathcal{M}: \Sigma_{g} \rightarrow \mathbb{C}$ is given by:

$$
\mathcal{Y} \mathcal{M}(\alpha)=\frac{\omega}{d} \operatorname{tr}(\phi(\alpha))
$$

Proof. From [24] the map $\phi$ is injective and onto. Hence we can identify $K_{r, f}\left(\Sigma_{g}\right)$ with $\operatorname{End}\left(K_{r, f}\left(H_{g}\right)\right)$. The Yang-Mills measure is zero on commutators. Thus it factors through

$$
\operatorname{End}\left(K_{r, f}\left(H_{g}\right)\right) /\left[\operatorname{End}\left(K_{r, f}\left(H_{g}\right)\right), \operatorname{End}\left(K_{r, f}\left(H_{g}\right)\right)\right] .
$$

This quotient is a 1-dimensional vector space. Hence any two linear functionals that factor through this quotient are equal if they agree on the identity matrix. The trace also vanishes on commutators, thus it factors through the commutator quotient. The normalization in the formula causes the two induced linear functionals to be the same.

Next we address the cases of $t= \pm 1$. Since the formula for the measure of a skein represented by a colored framed trivalent spine is an even function of $t$, we only need to consider one value. The value $t=-1$ is more convenient as the correspondence between $K_{-1}(F)$ and the $S U(2)$-characters of $\pi_{1}(F)$ is simpler. The skein of the disjoint union of curves $c_{i}$ corresponds to the function that sends the representation $\rho$ to

$$
\prod_{i}-\operatorname{tr}\left(\rho\left(c_{i}\right)\right)
$$


Theorem 4. The Yang-Mills measure is well defined on $K_{ \pm 1}\left(\Sigma_{g}\right)$ for $g>1$. Let $s_{c}$ be the skein in $K_{t}\left(\Sigma_{g}\right)$ corresponding to an admissibly colored trivalent spine of $\Sigma_{g, 1}$. If $t_{n}$, with $\left|t_{n}\right| \neq 1$, is a sequence of complex numbers converging to \pm 1 then

$$
\lim _{n \rightarrow \infty} \mathcal{Y} \mathcal{M}_{t_{n}}\left(s_{c}\right)=\mathcal{Y} \mathcal{M}_{ \pm 1}\left(s_{c}\right)
$$

Proof. Recall (23) that the measure of a skein $s_{c}$ in $K_{t_{n}}\left(\Sigma_{g}\right)$ is given by

$$
\mathcal{Y} \mathcal{M}_{t_{n}}\left(s_{c}\right)=\lim _{k \rightarrow \infty} \sum_{i=0}^{k}(-1)^{i}[i+1] \prod_{j=1}^{6 g-3} \frac{1}{\theta\left(i, i, k_{j}\right)} \prod_{v} \operatorname{Tet}\left(\begin{array}{ccc}
i & i & i \\
k_{v 1} & k_{v 2} & k_{v 3}
\end{array}\right) \text {, }
$$

and $\mathcal{Y} \mathcal{M}_{-1}\left(s_{c}\right)$ is given by the same formula as (44) except that quantized integers are replaced by ordinary integers in all the quantities involved.

The quantities for working with skeins in $K_{-1}(F)$ are the same as the ones for $|t| \neq 1$ except that quantized integers are replaced by ordinary integers. These formulas are the limits as $t \rightarrow-1$ of the values we have been using. Revisiting the fundamental estimate (20), we see that,

$$
\frac{\left|\operatorname{Tet}\left(\begin{array}{ccc}
i & i & i \\
k_{1} & k_{2} & k_{3}
\end{array}\right)\right|}{\sqrt{\left|\theta\left(i, i, k_{1}\right) \theta\left(i, i, k_{2}\right) \theta\left(i, i, k_{3}\right)\right|}} \leq \sqrt{\frac{\theta\left(k_{1}, k_{2}, k_{3}\right)}{(-1)^{i+k_{3}}\left(k_{3}+1\right)(i+1)}}
$$

from which we conclude that the right hand side is less than or equal to

$$
\frac{C\left(k_{1}, k_{2}, k_{3}\right)}{\sqrt{i+1}} \text {. }
$$

Considering the series for the Yang-Mills measure of a spine, comparison to the $p$-series implies that it converges as long as the surface has genus greater than 1. Similarly, the Yang-Mills measure is invariant under handle-slides.

In order to prove the convergence statement (43) choose $\epsilon>0$. The fundamental estimate allows us to find $K$ such that, for any $t_{n}$ as well as for $t=-1$, the absolute value of the tail of the sum (44) starting with $i=K$ is less than $\epsilon / 4$. Since the terms of the series for $\mathcal{Y} \mathcal{M}_{t_{n}}\left(s_{c}\right)$ converge to the respective terms of the series for $\mathcal{Y} \mathcal{M}_{-1}\left(s_{c}\right)$ as $t_{n} \rightarrow-1$, there exists $\delta>0$ such that if $\left|t_{n}+1\right|<\delta$ then the absolute value of the difference of the first $K$ terms of both series is less than $\epsilon / 2$. Finally, choose $N$ so that $n \geq N$ implies that $\left|t_{n}+1\right|<\delta$, and use the triangle inequality to see that

$$
\left|\mathcal{Y} \mathcal{M}_{t_{n}}\left(s_{c}\right)-\mathcal{Y} \mathcal{M}_{-1}\left(s_{c}\right)\right|<\epsilon
$$

for $n \geq N$.

For a surface of genus 1 we divide the partial sums, as before, by the number of terms in the sum, and the series then converges.

Theorem 5. The Yang-Mills measure at $t=-1$ is the symplectic measure on $\mathcal{M}\left(\Sigma_{g}\right)$. 
Proof. Using Weyl orthogonality to compute Witten's Yang-Mills measure for a surface of area $\rho$ yields that its value on the spine $s_{c}$ is given by the series

$$
\sum_{i=0}^{\infty}(-1)^{i}(i+1) e^{-\rho c_{2}(i)} \prod_{j=1}^{6 g-3} \frac{1}{\theta\left(i, i, k_{j}\right)} \prod_{v} \operatorname{Tet}\left(\begin{array}{ccc}
i & i & i \\
k_{v 1} & k_{v 2} & k_{v 3}
\end{array}\right),
$$

where the edges of $s_{c}$ carry colors $k_{i}$, and $k_{v_{i}}$ are the colors of the edges ending at the vertex $v$, and $c_{2}(i)$ is the value of the quadratic Casimir operator on the $(i+1)$-dimensional irreducible representation of $S U(2)$. As both Witten's series and our series converge absolutely, and Witten's formula converges term by term to our formula as $\rho \rightarrow 0$, the limit of Witten's Yang-Mills measure is equal to our Yang-Mills measure at $t=-1$. Finally, Forman [7] showed that the limit as $\rho \rightarrow 0$ of Witten's measure is the symplectic measure on $\mathcal{M}\left(\Sigma_{g}\right)$, normalized as in [7].

Suppose now that $|t|=1$ and $t$ is not a root of unity. Evaluation of the YangMills measure on the empty skein on a surface of genus $g$ yields $\sum_{i=0}^{\infty} \frac{1}{[i+1]^{2 g-2}}$. As $t$ is not a root of unity the number $[i+1]^{2 g-2}$ gets arbitrarily close to 1 infinitely often, which means that the series does not converge. Therefore the Yang-Mills measure does not exist away from roots of unity on the unit circle.

\section{References}

[1] A. Y. Alekseev, H. Grosse and V. Schomerus, Combinatorial Quantization of the Hamiltonian Chern-Simons Theory I, II, Comm. Math. Phys. 172 (1995), no. 2, 317-358, and Comm. Math. Phys. 174 (1996), no. 3, 561-604.

[2] D. Bullock, The $(2, \infty)$-skein module of the complement of a $(2,2 p+1)$ torus knot, J. Knot Theory Ramifications, 4 (1995), no. 4, 619-632.

[3] D. Bullock, C. Frohman and J. Kania-Bartoszyńska, Understanding the Kauffman bracket skein module, JKTR, 8 (1999), 265-277.

[4] D. Bullock, C. Frohman and J. Kania-Bartoszyńska, Topological interpretations of Lattice Gauge Field Theory, Comm. Math. Phys. 198 (1998), 47-81.

[5] D. Bullock, C. Frohman, J. Kania-Bartoszyńska, The Kauffman Bracket Skein as an Algebra of Observables, PAMS 130 (2002), 2479-2485.

[6] E. Buffenoir and Ph. Roche, Two Dimensional Lattice Gauge Field Theory Based on a Quantum Group, Comm. Math. Phys. 170 (1995), 669-698.

[7] R. Forman, Small volume limits of 2-d Yang-Mills, Comm. Math. Phys. 151 (1993), no. 1, $39-52$.

[8] C. Frohman and R. Gelca, Skein modules and the noncommutative torus, Transactions of the AMS 352 (2000), 4877-4888.

[9] C. Frohman and J. Kania-Bartoszyńska, A Quantum Obstruction to Embedding, Math. Proc. of the Cambridge Philosophical Society 131 (2001), 279-293.

[10] C. Frohman and J. Kania-Bartoszyńska, Shadow World Evaluation of the Yang-Mills measure, preprint.

[11] W. M. Goldman, The symplectic nature of fundamental groups of surfaces, Advances in Mathematics 54 (1984), no. 2, 200-225.

[12] W. M. Goldman, Invariant functions on Lie groups and Hamiltonian flows of surface group representations, Invent. Math. 85 (1986), no. 2, 263-302. 
[13] J. Hoste and J. Przytycki, The Kauffman bracket skein module of $S^{1} \times S^{2}$, Mathematische Zeitschrift 220 (1995), no. 1, 65-73.

[14] L. H. Kauffman and S. Lins, Temperley-Lieb recoupling theory and invariants of 3manifolds, Ann. of Math. Studies 143, Princeton University Press (1994).

[15] R. Lawrence and D. Zagier, Modular forms and quantum invariants of 3-manifolds, in: Sir Michael Atiyah: a great mathematician of the twentieth century, Asian J. Math. 3, (1999), 93-107.

[16] W. B. R. Lickorish, An Introduction to Knot Theory, Springer, GTM 175, 1997.

[17] M. McLendon, personal communication.

[18] T. Le, H. Murakami, J. Murakami and T. Ohtsuki, A three-manifold invariant via the Kontsevich integral, Osaka J. Math. 36 (1999), no. 2, 365-39.

[19] J. H. Przytycki, Fundamentals of Kauffman Bracket Skein Modules, Kobe Math. J. 16, (1999) 45-66.

[20] J. H. Przytycki, Kauffman bracket skein module of a connected sum of 3-manifolds, Manuscripta Math. 101 (2000), 199-207.

[21] N. Y. Reshetikhin and V. G. Turaev, Invariants of 3-manifolds via link polynomials and quantum groups, Invent. Math. 103 (1991), 547-597.

[22] J. Roberts, Skein theories as TQFTs, preprint.

[23] J. Roberts, Skein theory and Turaev-Viro invariants, Topology 34 (1995), 771-787.

[24] J. Roberts, Quantum Invariants via Skein Theory, Thesis, Pembroke College, Cambridge, 1994.

[25] V. G. Turaev, Shadow links and face models of statistical mechanics, J. Differential Geom. 36 (1992), no. 1, 35-74.

[26] H. Wenzl, On sequences of projectors, C.R. Math. Rep. Acad. Sci. IX (1987), 5-9.

[27] E. Witten, Quantum field theory and the Jones polynomial, Comm. Math. Phys. 121 (1989), 351-399.

[28] E. Witten, Quantum Gauge Theories in Dimension Two, Comm. Math. Phys. 141 (1991), 153-209.

D. Bullock

Department of Mathematics

Boise State University

Boise, ID 83725

USA

e-mail: bullock@math.boisestate.edu

Joanna Kania-Bartoszynska

Department of Mathematics

Boise State University

Boise, ID 83725

USA

e-mail: kania@math.boisestate.edu

(Received: June 2, 2000)
C. Frohman

Department of Mathematics

University of Iowa

Iowa City, IA 52242

USA

e-mail: frohman@math.uiowa.edu

\section{(7) To access this journal online: \\ (4) http://www.birkhauser.ch}

